\title{
The Chinese Game Room: Play, Productivity, and Computing at Their Limits
}

\author{
Julian Dibbell, \\ Contributing editor, Wired magazine, and author of Play Money: Or How I Quit My Day Job and Made \\ Millions Trading Virtual Loot
}

Keywords: China, video games, artificial intelligence, political economy, ludocapitalism

\section{1}

There is a room in China: It is a big room, a former manufacturing space now filled with rows of networked computers, maybe 50 in all. Young men sit gazing at the computer screens: pointing, clicking, typing, earning a living.

They are paid to play a game - the massively multiplayer online role-playing game World of Warcraft- and they play it steadily. In daily 12-hour shifts, they run their characters through WoW's complex fantasy universe, killing monsters, gathering gold coins and magic weapons from the monsters' corpses, selling the weapons to other players for yet more gold coins. These coins, as well as the experience points their characters accrue, are the fruits of the young men's labor, for which they are paid about 30 yuan per day, or roughly US\$0.25 an hour. Their employers sell these commodities at twice that rate or more to online retailers, who eventually, in turn, sell them to the end buyers: World of Warcraft players in North America and Europe who are happy to pay real money for the means to get ahead in the game. These players are generally as ignorant as any Nike customer of the industrial conditions in which the objects of their desire are produced, with this one difference: It is not at all unlikely that a Western player will, on any given day, cross paths with a Chinese worker inside the game's bright, colorful world and even exchange a few words with him friendly or otherwise, aware, or not, that the person at the other end of the conversation sits in a large factory room on the far side of the world.

I have seen this room myself, with my own eyes. It is on the second floor of a nondescript factory building in a commercial district of the midsize city of Jinhua, which I visited in $2006 .{ }^{1}$ It is hardly the only such room in China. There are as many as 100,000 operations like it there, I was told. They are known as gold farms, and the market they supply is for what's known as realmoney trading, or RMT.

In one form or another, RMT exists in nearly every one of the world's massively multiplayer online games - or MMOs - which range from the 11-million-player
World of Warcraft down through second-tier hits like Lineage and Final Fantasy and into a long tail of cult and boutique games like EVE and Iron Realms and $A$ Tale in the Desert, all of them providing a mix of fantasy play and social interaction whose appeal, for its enthusiasts, verges on the addictive. An estimated 30 million people, worldwide, play these games. The companies that produce them earn most of their revenue from subscription fees, and very little of it from RMT, which overwhelmingly involves trade between one customer and another and in most MMOs, in fact, directly violates the rules. It thrives nonetheless. By the latest estimates, the amount of real money annually exchanged for virtual commodities now rivals the total revenues of the MMO producers themselves: about US\$2 billion. ${ }^{2}$

And even that figure, viewed from a more rigorously econometric perspective, understates by as much as an order of magnitude the total wealth produced by virtual economies. After all, for every gold coin or magic sword that is wrested from the monsterridden wildernesses of these games and traded for real money, many more trade only for in-game goods, or not at all. A World of Warcraft player whose character has spent an evening wandering the virtual countryside in search of rare and potent herbs might choose, at the end of the night, to sell her harvest to other players for a few gold coins, or she might choose, instead, to use the herbs herself in making powerful magic potions for her next encounter with the dragon she is bent on slaying. But either way, an economist familiar with the RMT markets can easily assign a real-money value to the outcome of the player's herb gathering. And given enough comparable data points, the economist could even put a number on the aggregate value of all goods thus brought into World of Warcraft's virtual economy in a given year. In short, he could calculate the game's gross domestic product.

In 2001, the economist Edward Castronova published just such a calculation for EverQuest, the most popular MMO at the time: The GDP of EverQuest, he determined, was US\$135 million. ${ }^{3}$ Updated and more broadly applied, Castronova's methods point to a total GDP for all existing virtual economies of over US\$28 billion - comparable to the gross domestic product of Lithuania or Sri Lanka. ${ }^{4}$ 
Most of that wealth is created by unpaid players, playing, and this of course is not at all how production usually happens in conventional economic modernity. Indeed, so antithetical are play and production in contemporary common sense that it is tempting to dismiss a formulation like Castronova's as itself merely playful - as a winking parody or arch perversion of the classical econometric paradigm rather than an invitation to rethink the conceptual foundations on which that paradigm rests. As someone who has been teaching and popularizing Castronova's work for years, I can attest to the eagerness with which lay readers seek to find a flaw in his analysis, some catch that shows it all to be a joke and leaves the fundamental distinction between work and play undisturbed. And I confess that at the time I got the opportunity to go to China, I too still doubted whether the loopy, boundary-crossing economics of MMOs could really be doing much more than wrinkling that distinction. By then I had also been playing World of Warcraft steadily - and at times obsessively - for nearly a year, so I was intimately familiar with the well-documented arduousness of MMO play. For all it felt like labor, however, I don't think I ever imagined that a first-hand glimpse of the gold farms, where WoW's laboriousness arrived at the limit case of literal wage work, would fail to show me some essential difference between the farmers' productivity and mine. I went to China, therefore, expecting to return with a better understanding of what drew the line between work and play. I thought, in any event, that the line itself would be easy to see.

I was wrong. The end of the first day's shift at Jinhua should have been as clear a line as I could have asked for. The clock struck 8 p.m.; the workers rose from their seats, they punched their time cards, and for the first time in 12 hours they were free from the compulsory gaming that had consumed their day. What would these young men do now with their precious few moments of free time? How would they amuse themselves? I followed them out of the room and was not surprised to see that some retired to their company dorm rooms for idle conversation while others sat in the break room watching television. But quite a few of them, it turned out - nearly half headed straight to a nearby Internet café to spend the evening doing exactly what their job had required them to do all day: play World of Warcraft. And this I was at a loss to account for.

It might be said, of course, that there was no puzzle here - that in fact the defining difference between gaming as work and gaming as play was laid out plain as day before me. It might be said, that is, that what those off-duty gold farmers in that Internet café were doing was not at all the exact same thing they'd been doing on the job but in a sense its precise opposite. Instead of playing a game for other people and for other ends - for their bosses, for their wages - they were playing it now for themselves, on their own terms and as an end in itself. But the distinction, while valid, only deepens the mystery. Among the defining hallmarks of modern, alienated labor, Marx observed, is "that as soon as no physical or other compulsion exists, it is shunned like the plague", ${ }^{5}$ and it is puzzling indeed to find that the "alienated play" 6 of the gold farms should have so strikingly different an effect. Self-directed or not, the after-hours play of the gold farmers brought them back, night after night, to what was in effect their work site, and I found it hard to believe they would choose this if the job itself were simply a job.

Indeed, when I returned the next day to watch those same men back at work, I found myself noticing an interesting thing about all that alienated play on the shop floor: It actually looked, here and there, rather playful. There sat 23-year-old Xu Xuidong, for instance, taking a World of Warcraft gnome character into battle while behind him 26-year-old Shao Meizhong stood watching. The gnome died, Shao laughed, and when he reached down to the keyboard to demonstrate a more effective combination of combat spells, there was an eagerness in the gesture that suggested Shao would not mind sitting down for a turn himself. It was a scene repeated in one form or another throughout the room and throughout the shift, one worker breaking briefly out of the prevailing solitude to watch another's progress, argue strategies with him, cheer him on.

Not that they were in love with their jobs. After six months, Xu had had his fill: "The shifts are too long, your eyes hurt... it isn't so interesting any more." ${ }^{7}$ Nor was Xu among those workers who wanted anything to do with video games after his shift ended. But even so, he admitted, there remained those working moments when he was in a close fight with a virtual monster, uncertain which of them would win, and he would find his heart racing, his adrenalin pumping, his whole focus brought to bear on the game.

"I do. I still feel excitement", Xu said. He seemed to be struggling as much as I was to understand how a simple game of make-believe mortal combat, even under the crushing weight of routinization that his workday brought to bear on it, could retain its power to engage him. Then he shrugged, as if perhaps it didn't really need a lot of explaining: "We don't want to die", he said.

And that was when it sank in finally that, whatever vast cultural and economic gaps stood between me and the gold farmers, I would find here no essential difference between their relationship to the game and mine. Nor was that factory room in Jinhua, perhaps, the only case that might illuminate the productive play we found ourselves engaged in.

\section{2}

Let us consider, then, another room. It might be in China, or it might not. Certainly there is a lot of Chinese conversation going on inside it, of a sort. The room is occupied by one person, who has access to paper, pencils, and one very large reference book. The door to the room is closed, but now and then somebody slips a piece of paper under the door with messages written on it in Chinese characters. The person in the room cannot read or understand a word of Chinese, but he has the reference book, and 
it contains an elaborate set of rules for answering whatever messages arrive. So sophisticated and comprehensive is the rule set, in fact, that simply by looking up the Chinese characters given to him and then writing out the characters the rules dictate as responses, he can convince any Chinese speaker standing outside the door that there is, inside, an equally fluent Chinese speaker capable of intelligent conversation.

There are similarities (some obvious, some less so) between this Chinese room and the one I visited in Jinhua in 2006, but the principal difference is this: This room has never existed except as an intellectual exercise. It is the central conceit in an argument crafted by the philosopher John Searle and published first in $1980 .{ }^{8}$ Much debated and now generally referred to as the Chinese Room argument, this was Searle's response to the earlier, and still somewhat better known, thought experiment now known as the Turing test.

The Turing test was dreamed up by computing's foundational theorist, Alan Turing, as a way to reframe what, by the late 1940s, had already become a tiresomely intractable question about the new digital machines: Could they think? Or might they someday be able to ? $^{9}$ Turing's bold stroke was to scrap the question altogether and replace it with a party game. His starting point was an amusement known as the Imitation Game, in which two party guests, one male and one female, would sit hidden in separate rooms and take questions about their gender from the other guests, each competing to convince the questioners that he, or she, was the woman. Turing, though, proposed a variation: Take a computer programmed for what nowadays would be called natural-language processing, put it in a room with a reasonably intelligent human being, and put a human "judge" in a neighboring room connected to the first by a teletype line. Then, let the judge have open-ended written exchanges with both the human and the computer. If in more than $50 \%$ of these conversations the judge fails correctly to guess which correspondent is the human, then the machine has passed the test and, whether or not we grant it anything like a soul, must be said for all practical purposes to be doing all the thinking we require of most fellow human beings anyway.

As rhetorical gambits go, the Turing test may be a cheap trick, but it is an effective one, and it has stood up nicely over the years against a range of obvious metaphysical and phenomenological objections. One counterargument has proved a formidable match, however, and that is Searle's, which succeeds in large part because it takes the same rhetorical strategy that worked so well for Turing and turns it exactly on its head.

Searle begins where Turing does, granting the assumption that a piece of software will someday be written that can engage in robust verbal communication with intelligent people. Instead of picturing the software coded into a computer, though, Searle imagines it written into a book - the reference book that the resident of the Chinese Room uses to guide his communications with the Chinese speakers outside the room. The result, for those outsiders, is just what it was for the judge in the successful Turing test: a seamlessly convincing conversation. But theirs is not the point of view that interests Searle. What he wants to know about is the person on the inside. Can that human natural-language processor be said, by any stretch of logic, to actually understand the language he is processing? And if this manifestly sentient individual does not in fact come close to grasping what that processed stream of Chinese characters means, can a digital processor possibly come any closer? The answer, for Searle and for those who buy his argument, is a crisp, clear no.

For those who do not buy it, on the other hand and the number of cognitive scientists, computer researchers, systems theorists, and others that have weighed in against Searle's position over the years has grown to an impressive crowd - the response, at its core, is equally clear: Why should we care at all what the person inside the Chinese Room does or does not understand? If the entire room, including not just its occupant but the vastly complicated rule book he works from, delivers Chinese speech as fluent as any spoken by a human, why look for understanding in just one piece of the room's machinery? Why not ask, as well, if a single neuron in the brain of a Chinese speaker understands the language it assists in uttering?

\section{3}

Let us leave these questions aside for the moment, though, and turn to one I am mildly surprised that nobody, it seems, has ever thought to ask of Searle and his thought experiment: What exactly is that person doing inside the Chinese Room in the first place? What motivates her to be there and perform the tasks required?

The question is hardly central to Searle's argument, of course, but it is worth noting that Turing, with his party-game scenario, did not exactly leave it unaddressed in setting up his own thought experiment. And while there are various answers that could be proposed in Searle's case - the Chinese Room's inhabitant might be a researcher personally interested in the outcome of the experiment, for instance, or a paid functionary simply trying to earn a living - the more I have thought about this, the more I think the answer that is both the likeliest and the most useful is the same one Turing proposed: The person in the Chinese Room is doing it for fun.

That may seem doubtful to you, but if you have spent as much time as I have playing games like World of Warcraft, you will realize that the experience of operating the Chinese Room might not be all that different. MMOs may be dressed up in the clothes of fantasy and adventure, but at their heart they are really just very complex yet very mechanical systems of inputs, lookup tables, and outputs - no different in that regard from any computer program, of course, but different (and with regard to the Chinese Room, 
uniquely similar) in the sense that they put a human actor in the position of managing the flow of data into and through the lookup tables.

Could the rule set of the Chinese Room actually be designed in such a way that its inhabitant would experience it as a game while its "users" - the Chinese speakers on the outside, interacting with it - would experience it as productive of coherent conversation? Why not? The genius, and the usefulness, of both Turing's and Searle's scenarios lies in their indifference to any existing computer technology and their focus, instead, on the theoretical limits of computing, as established early on by Turing himself. What they illuminate is not computers as we know them, but the computer as Turing first, axiomatically, described it: as a "universal machine", a system capable of enacting in code the workings of any other system, physical or logical, knowable or imaginable. In particular, the Turing test and the Chinese Room draw out the implications of that axiom's most unsettling corollary: that computers can in principle reproduce the workings of the human brain.

Yet there are other corollaries worth attending to, and one of them is that, for any two systems simulated on a given Turing machine, a third program can be written that recodes the outputs of one as meaningful inputs for the other, and vice versa. Think of Borges's "Library of Babel", which contains every possible volume that the letters of the alphabet can compose, and in which there are no two books that some third book or set of books, a lexicon, does not prove to be perfect translations of one another. The principle is similar, and the implication it points us to is this: Any productive process - any job, in short - that can be rendered as a computer program requiring human input can also, theoretically, be designed to take that input via a program humans might want to play.

Nor is the proposition quite so purely theoretical. Games scholar Nicholas Yee, thinking through the repetitive, laborious nature of many $\mathrm{MMOs}$, has proposed half-seriously that the current practice among Western radiologists of transmitting digitized $X$-rays to India for low-cost overnight analysis could be replaced by an even cheaper method: design a sci-fi MMO in which training up a skill in, say, "pattern recognition" would involve clicking on anomalies in actual X-rays submitted to the players for analysis. ${ }^{10}$ Or consider the already existing Google Image Labeler. Based on work by computer scientist Luis von Ahn - a founding researcher in the growing field of "games with a purpose" - Image Labeler is an addictive little game that challenges players to tag images with common-sense labels ("blonde", "woman", "singer", for, e.g., a Cristina Aguilera photo), thus turning their idle play into what, for Google's purposes, is productive labor.

These may be trivial examples, but the development they point to is anything but: At its peripheries and at its core, the world's economy appears to be waking up to the interesting fact that play can be productive - and that digital environments can be especially effective in channeling play toward productivity.

I have argued elsewhere that this emergent mode of production - ludocapitalism, as I have taken to calling it - is a trend of potentially sweeping dimensions, and I am not alone in this. In his sharply reasoned book Hacking Capitalism, ${ }^{11}$ Marxist cultural scholar Johan Söderberg gives intellectual force to an argument that has long percolated in the margins of the free and open-source software movement: That the economic mystery of what motivates FOSS coders to volunteer their efforts to the development of Linux, Apache, and other pivotally productive programs is in fact no mystery at all. They do not do it out of a sense of altruism or political commitment or any of the other forced explanations analysts have offered. They do it, rather, for a very complex sort of fun. It is play that drives their economic productivity, and if we are lucky, Söderberg argues, the productive dynamics of FOSS will radiate throughout the economy so that play, eventually, will drive and liberate us all. It is not that Söderberg does not recognize the ways in which, even before the spread of digital technologies, capitalism had already begun sniffing out ways to exploit the energies of play. But he is confident that, properly suffused with the power of production, the "aesthetic play-drive" (as the late eighteenth-century political philosophy of Friedrich Schiller celebrated it) will in the end prove fatally resistant to capitalism's alienating logic.

Myself, I'm not so sure. The richly social and impassioned world of FOSS production may be fertile ground for optimism like Söderberg's, but when I think of the Jinhua factory room I visited - where textbook capitalist exploitation thrives in peaceful and productive coexistence with the play-drive of the exploited - I have a hard time sharing that optimism. Not that pessimism is the alternative I propose. What I offer, instead, is this other, more abstract Chinese room: Searle's imaginary chamber reconfigured and repurposed, finally, as a tool for thinking about productive play at the limits of its possibilities. In the end, what is valuable about both Searle's and Turing's experiments is not whether they prove or disprove the proposition that machines can thinkindeed, both fail resoundingly on that account - but how they sharpen our sense of what that proposition possibly could mean, both to our relationships with our machines and to our relationships with thought itself. Similarly, mine takes for granted what might otherwise seem most in need of proving - that machines could someday turn even the most complex of jobs into a game, universally converting the energy of play into the force of production - and asks instead that we consider what that proposition means, both to our relationship to work and to what work might become. Does it portend the final subjugation of play to capitalism's rationalizing demands, or the contrary? Does it call us to remake our productive lives in its image, or to resist it? 
In short, this Chinese room of mine asks no more than that we take the notion of productive play seriously enough to imagine a world overrun with it.

\section{4}

I have not forgotten, of course, that this is asking a lot - that modern common wisdom holds productive play itself to be a contradiction in terms, and that it beggars conventional logic, therefore, even to conceive a world in which games do all the real work. I am aware, too, that this wisdom has a formidable intellectual pedigree, anchored as it is in the founding, twentieth-century texts of game studies - in Johan Huizinga's Homo Ludens and more emphatically in Roger Caillois's Man, Play, and Games, where play is famously defined as "an occasion of pure waste".

Nor would I quite so confidently insist that this logic has outlived its relevance were it not for later students of play and games (Brian Sutton-Smith, ${ }^{12}$ Celia Pearce ${ }_{1}^{13}$ Thomas Malaby, ${ }_{1}^{14}$ and others) who have worked hard to problematize the definition of play as an inherently frivolous and unproductive act. Indeed, so problematic has this conflation of the ludic and the futile been shown to be - so parochially modern and Western and yet so deeply threaded into our everyday thinking about play - that it is tempting to set the notion of play aside altogether when addressing the place of games in the contemporary world. This appears to be the strategy preferred by Malaby, for instance, who proposes a definition of games as "domains of contrived contingency", 15 separating "play" out as a subjective, psychological state or attitude that may or may not be present in the unfolding of a given game.

And yet, while this play-agnostic approach may do the evolving field of game studies a world of epistemological good, it seems finally not a much more useful approach to the evolving relationship between work and games than the assumption that that relationship can only be one of mutual antithesis. For again, just as the question posed by Turing and Searle's thought experiments was not so much "Can machines think?" as "Does it matter whether and how we understand them to be thinking?", what is at stake here is not the formal capabilities of games so much as, precisely, the subjective, psychological states and attitudes they conjure in us. The question posed by my own thought experiment, in other words - and by the accelerating convergence of games, computers, and productivity generally - is not "Can machines turn work into games?" but "Does it matter whether and why we want to play those games?"

The answer, crucially, is yes. It does. It matters first and foremost for the crudely obvious reason that play, as an attitude, tends to get people doing things without their getting paid to do them. From the perspective of capital, in fact, it matters for no other reason whatsoever. Extracting surplus value from productive human activity is what capital does, after all, and any technique that lowers the market price of that activity is a technique that capital will, all else being equal, pursue with vigor. Even if Söderberg is right, then, about the long-term incompatibility of capitalism and the play-drive, a capitalism that in the near term has figured out a way to make the playdrive work for it is hardly likely to concede the point without a fight.

And so, if for no other reason, it matters also to us productive humans whether and why we want to play the games that do the work. Nor do I think we can meaningfully answer these questions without understanding play and games to be, if not logically inseparable entities, then integral and intimately coupled elements of a single, historically emergent system - which itself is fused with, on the one hand, the digital technologies that now almost define our games and, on the other, the political economy that feeds and, more and more, feeds on our gaming. That is not to say we cannot still usefully understand play to be an essentially subjective phenomenon. On the contrary, play's subjectivity is central to this view: It is precisely what draws us and embeds us, as desiring, playing subjects, into this broader, ludic system. But it is also, therefore, what is making play less and less a traditionally human phenomenon and more and more a cyborg one.

If I have linked the question of productive play to the rhetorical strategies of Searle's Chinese Room, then, that is not just for rhetorical reasons. It is ultimately because that question is of a piece with those that Searle's argument, like Turing's, pursues: They are questions of human identity in the age of the universal machine. Searle's Chinese Room imagines human subjectivity spliced into that machine in hopes of thereby proving the uniqueness of human thought; mine imagines the same thing in hopes of locating the productive limits of human play. But both are called to their task by the historical fact that the fusion of humanity and its universal machine has long since ceased to be entirely imaginary.

For what it is worth, this may not be as dehumanizing a development as it seems. As the anthropologist Eduardo Viveiros de Castro has remarked, Karl Marx can be read to define Homo sapiens as "the universal animal"16 in much the same way Turing defines the computer as the universal machine, and if that definition holds any truth then our cyborg trajectory might promise not so much a loss as an expansion of human identity. What is perhaps more interesting, however, is that Marx locates our essential universality not in human thought, with its seemingly endless versatility, but in the equally expansive versatility of human production:

In creating a world of objects by his practical activity, in his work upon inorganic nature, man proves himself a conscious species being .... Admittedly animals also produce. They build themselves nests, dwellings, like the bees, beavers, ants, etc. But an animal only produces what it immediately needs for itself or its young. It produces one-sidedly, whilst man produces universally. It produces under the dominion of immediate physical need, whilst man produces even when he is free from physical need and only 
truly produces in freedom therefrom. An animal produces only itself, whilst man reproduces the whole of nature. ${ }^{17}$

This passage, by the way, is from Marx's famous essay on "Estranged Labor", the same one whose remarks on the leisure pursuits of the alienated worker so dramatically conflicted with the afterhours gaming I observed among the hard-worked gold farmers of China. But in these lines on the nature of human production there is more resonance than not with what I saw in Jinhua. What leaps out here, to the eye that has already seen how intricately the ludic and the laborious can intertwine, is the repeated implication that however useful any given human labor may be, it is only genuinely human insofar as it is effectively unnecessary. A human being "only truly produces" in freedom from necessity, conscious that whatever productive act she commits to is only ever one among the infinite variety available to the universal animal. And while, as a description of what most of us know as work, this is a generally remote ideal, we regularly encounter something not a lot unlike it in the routine needlessness of play.

Let me now repeat: My memories of the gold farms are still too vivid for me to think that simply flooding our productive lives with play will suffice to free us from even the crudest varieties of economic exploitation. I shall only say that the very least we can do for productive players present and future for the gamers at work in China today and for those who inhabit whatever "Chinese rooms" may come - is to keep pursuing as full an understanding of the relationship between play and production as we can. That play cannot be productive is easy enough to recognize now as fallacy. But perhaps it is not even enough to acknowledge that play can be productive. What it may be time to explore - what the coevolution of the universal animal and the universal machine may ultimately compel us to explore - is the possibility that production, in its truest sense, is play.

\section{NOTES}

1. I went to China on assignment from the New York Times Magazine, researching an article published later, in the magazine's June 17, 2007, issue, as "The Life of the Chinese Gold Farmer". Throughout the trip I had the invaluable assistance of $\mathrm{Ge}$ Jin, a Shanghai native who, at the time, was working on a documentary about the gaming factories as a project in the UC San Diego graduate communications program.

2. Leihtiniemi, Tuukka. "How Big Is the RMT Market Anyway?" Virtual Economy Research Network. March 2, 2007. Retrieved February 6, 2008, from http://www.virtual-economy.org/blog/how_big_is_ the_rmt_market_anyw

3. Castronova, Edward. Virtual Worlds: A First-Hand Account of Market and Society on the Cyberian Frontier. Social Science Research Network. December 2001. Retrieved February 6, 2008, from http://ssrn.com/abstract=294828
4. Dibbell, Julian. Recalculating the Global Virtual GDP, Yet Again. Terra Nova. June 26, 2007. Retrieved February 6, 2008, from http://terranova.blogs.com/ terra_nova/2007/06/recalculating-t.html

5. Marx, Karl (1969). Economic and Philosophical Manuscripts of 1844 (p. 111). New York: International Publishers.

6. I have borrowed the term from $\mathrm{Ge}$ Jin, who used it to describe the gold farmers' on-the-job play in a talk given at University of California, San Diego, February $8,2007$.

7. In this and all my conversations with the gold farmers, Ge Jin served as interpreter.

8. Searle, John (1980). Minds, Brains and Programs. Behavioral and Brain Sciences, 3 (3), 417-457.

9. Turing, Alan (1950, October). Computing Machinery and Intelligence. Mind, LIX(236), 433-460.

10. Yee, Nick. The Blurring of Work and Play. The Daedalus Project. July 9, 2004. Retrieved February 6, 2008, from http://www.nickyee.com/daedalus/ archives/print/000819.php

11. Söderberg, Johan (2007). Hacking Capitalism: The Free and Open Source Software Movement. New York: Routledge.

12. Sutton-Smith, Brian (1997). The Ambiguity of Play. Cambridge, MA: Harvard University Press.

13. Pearce, Celia (2006). Productive play: Game culture from the ground up. Games and Culture, 1(1), 17-24.

14. Malaby, Thomas M. Anthropology and Play: The Contours of Playful Experience. Social Science Research Network. December 12, 2008. Retrieved February 6, 2008 from http://ssrn.com/ abstract $=1315542$

15. Malaby, Thomas M. (2007). Beyond Play: A New Approach to Games. Games and Culture, 2(2), 95-113.

16. Viveiros de Castro, Eduardo (2004). Exchanging Perspectives: The Transformation of Objects into Subjects in Amerindian Ontologies. Common Knowledge, 10(3), 463-484 (p. 467).

17. Marx, Karl (1964). The Economic and Philosophic Manuscripts of 1844, Ed. and intro. Dirk J. Struik. Trans. Martin Milligan. New York: International Publishers.

\section{CORRESPONDENCE}

Correspondence: Julian Dibbell.

E-mail: julian@juliandibbell.com

ISSN 1749-3463 print/ ISSN 1749-3471

DOI: $10.1080 / 17493460903020224$

(C) 2008 Artifact 